\title{
Frozen elephant trunk and hemiarch for type A aortic dissection
}

\author{
Saket Singh^, Sameh Yousef, Roland Assi, Prashanth Vallabhajosyula \\ Section of Cardiac Surgery, Yale School of Medicine, New Haven, CT, USA \\ Contributions: (I) Conception and design: All authors; (II) Administrative support: All authors; (III) Provision of study materials or patients: All \\ authors; (IV) Collection and assembly of data: All authors; (V) Data analysis and interpretation: All authors; (VI) Manuscript writing: All authors; (VII) \\ Final approval of manuscript: All authors. \\ Correspondence to: Prashanth Vallabhajosyula, MD, MS. Surgical Director of the Aortic Institute, Cardiac Surgery, Yale Cardiac Surgery, Yale \\ Physicians Building, 800 Howard Avenue, Ste 2nd Floor, New Haven, CT 06519, USA. Email: prashanth.vallabhajosyula@yale.edu.
}

\begin{abstract}
Type A aortic dissection (TAAD) is a surgical emergency, which has the potential to cause malperfusion in multiple organ systems. Perioperative morbidity and mortality, even in the most experienced centers, continues to be in double-digit. The natural history of patients who survive initial surgery is marred by distal aortic degeneration, which is responsible for decreased survival during midterm followup, significant late reintervention rate with associated morbidity and mortality, and also need for tighter surveillance during the follow-up. Aggressive hemiarch replacement with direct intimal repair and antegrade stent graft in the descending thoracic aorta (DTA) not only stabilizes the ascending aorta and aortic arch in the acute setting, it also retards continuous degeneration of the downstream aorta. In the immediate postoperative period, a stent graft in the DTA placed in an antegrade manner, eliminates the possibility of type 1a endoleak. Coverage of distal fenestration in DTA and true lumen stabilization with a rigid graft has the potential to mitigate malperfusion in this delicate patient population. In the long run, a stent graft in the DTA promotes aortic remodeling, provides a stable framework for future open/endovascular re-intervention. In this review article, we have detailed our operative approach in patients with TAAD, and discussed variables relevant for a good outcome.
\end{abstract}

Keywords: Type A dissection; frozen elephant trunk (FET); hemiarch; antegrade thoracic endovascular aortic repair (antegrade TEVAR)

Received: 20 March 2020; Accepted: 03 June 2020; Published: 20 October 2021.

doi: 10.21037 /jovs-20-85

View this article at: http://dx.doi.org/10.21037/jovs-20-85

\section{Introduction}

Type A aortic dissection (TAAD) is a surgical emergency that carries in-hospital mortality of $58 \%$ without surgical treatment (1). As per The International Registry of Acute Aortic Dissection (IRAD), surgical repair brings down in-hospital mortality to $18 \%$ (2). Surgical treatment of TAAD continues to evolve since the first report of successful repair of TAAD was published in $\mathcal{F} A M A$, in 1963 by DeBakey group (3). This success was based on years of experience with aortic surgery and research since DeBakey first described aortic arch aneurysm surgery in
1955 (4). According to GERAADA (German Registry for Acute Aortic Dissection Type A), 33.6\% of TAAD patients present with at least one organ malperfusion. There is an incremental increase in surgical mortality with the increasing number of malperfused organs (5). As per the IRAD database, mesenteric malperfusion increases inhospital surgical mortality to $41.7 \%$ after an open/hybrid approach. Persistent visceral malperfusion after conventional proximal aortic repair of TAAD is usually caused by entry tear in the distal arch or proximal descending aorta, causing flow in the false lumen and true lumen collapse. The group

$\wedge$ ORCID: 0000-0002-6419-6300. 


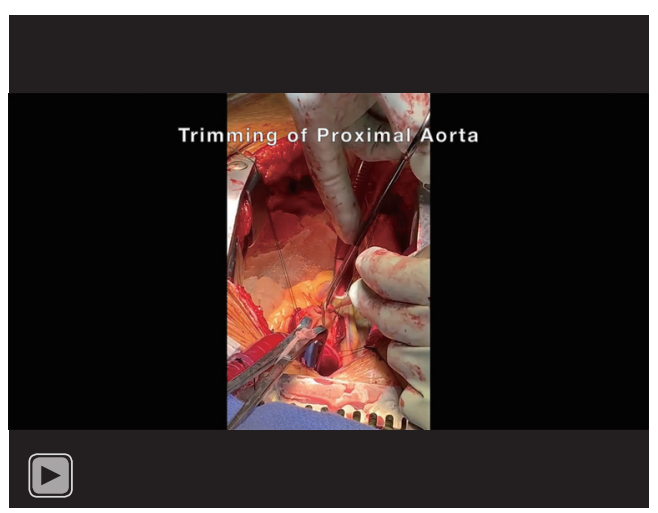

Video 1 This video demonstrates step by step hemiarch reconstruction and frozen elephant trunk repair of a patient with type A aortic dissection.

from Hannover, Germany first introduced frozen elephant trunk (FET) in 2003, in an attempt to seal this distal tear site and improve true lumen flow in the distal aorta. FET also promotes aortic remodeling by inducing thrombosis of the false lumen.

\section{Setup}

All patients with a diagnosis of TAAD are transported to the operating room in an emergent manner. CT scan images are reviewed and in addition to cannulation and perfusion strategy. CT Scan is also used to determine both the diameter and length of the stent-graft to be used in the descending thoracic aorta (DTA). Bilateral upper extremity arterial line and a Swan-Ganz catheter (Edwards Lifesciences, Irvine, USA) are used in all the patients. Upon induction, the diagnosis of TAAD is confirmed with transesophageal echocardiography. Cerebral oximetry and continuous EEG monitoring are used for neuromonitoring.

Aortic cannulation is mostly through direct true lumen cannulation of the ascending aorta/arch or axillary cannulation. The right atrium is cannulated using double stage venous cannula, which is connected through a Y-connector to single-stage right angle superior vena cava cannula. This single-stage venous cannula is connected to the cardioplegia line using a Y-connector. This cannula is used for perfusing oxygenated blood to the brain during hypothermic circulatory arrest (HCA). Presence of a normal-sized innominate vein is confirmed during initial dissection. Myocardial protection is achieved using antegrade cold blood cardioplegia (Video 1). A left ventricular vent is placed in all cases through the right superior pulmonary vein, and then systemic cooling is commenced. Proximal aortic work is done during the cooling period. Once electrocerebral silence is confirmed on EEG, patients are cooled to $3^{\circ}$ below this temperature and for 5 minutes beyond this landmark. EEG silence usually happens between 15 to 20 degrees, using a nasopharyngeal probe. The patient is put into a reverse Trendelenburg position, and HCA is instituted after stopping antegrade aortic perfusion. Superior vena cava is snared, and oxygenated normokalemic blood at $12{ }^{\circ} \mathrm{C}$ is perfused through the single-stage cannula connected to the cardioplegia line. Retrograde cerebral perfusion (RCP) pressure of $25 \mathrm{mmHg}$ and flow of $200-300 \mathrm{~mL} / \mathrm{min}$ is maintained. Adequate RCP is confirmed by dark blood draining out of brachiocephalic arteries ostium.

Aortic arch is resected in a steep oblique fashion starting from the origin of innominate on the greater curvature to the level of the isthmus on the lesser curvature. Distal arch reinforcement is done using Teflon felt neo-media (DuPont, Wilmington, DE) (Video 1). Proximal descending aorta and orifices of brachiocephalic arteries are then inspected. Any tear is primarily repaired with interrupted pledgeted "inside-out" sutures. A 0.035" Bentson wire (Cook Medical Inc., Bloomington, IN) is an advanced antegrade through the open arch into the DTA, and a pre-sized Relay Plus (Bolton Medical, Sunrise, FL) thoracic stent-graft system is introduced (Video 1). The proximal end of the stent is landed just distal to the left subclavian artery (LSA). After deployment, proximal end of the stent graft is then circumferentially secured to the aorta using interrupted pledgeted sutures. Hemiarch anastomosis is then completed using a Dacron tube graft (Vascutec Ltd., Renfrewshire, Scotland). The arch graft is cannulated, and the entire arterial system is deaired using the RCP circuit. The arch graft is then proximally clamped. RCP is terminated as antegrade flow through the arch cannula is started for the resumption of cardiopulmonary bypass and rewarming. At this time, the remaining proximal aortic/root work is completed. Cross clamp is then released. Once the patient is rewarmed, weaning from cardiopulmonary bypass is started.

\section{Discussion}

Hemiarch and FET in TAAD is still an evolving topic. It involves several contentious steps in this delicate patient population. 


\section{Neuromonitoring, cerebral perfusion, and deep bypothermia}

We confirm electrocerebral silence before initiation of HCA and RCP. Cannulation for CPB is adjusted, or arch fenestration is done if EEG changes are detected after the institution of $\mathrm{CPB}$.

Those with extensive arch reconstruction requiring more than 40 minutes of HCA, selective bilateral antegrade cerebral perfusion is used using balloon-tipped cannulas.

\section{Aortic root replacement}

We replace aortic root if the diameter of the aorta at the level of sinus of valsalva is greater than $45 \mathrm{~mm}$. Aortic root is also replaced if there is intimal tear involving the root, or in patients with aortic leaflet pathology.

\section{The diameter of the stent-graft}

Sizing is done based on CT scan findings. Diameter of the graft is $5 \%$ larger than the size of the true plus false lumen at the level of proximal DTA. We believe this gentle oversizing helps us prevent type Ib endoleak. This amount of oversizing, we believe, is also not aggressive enough to induce distal entry tears at the landing zone. We usually use stent-grafts between 37 to $40 \mathrm{~mm}$ in diameter.

\section{Length of the stent-graft}

We usually use a $150 \mathrm{~mm}$ stent graft. The aim is to reestablish true lumen flow, cover the distal entry site in DTA, achieve remodeling by inducing false lumen thrombosis, and avoid any future distal aortic reintervention.

There was no incidence of paraplegia using a $150 \mathrm{~mm}$ stent-graft in a paper published by one of the authors, in which this technique was used in 62 patients (6). In a series reported by Faure et al. (7), where the median length of stent-graft coverage was $200 \mathrm{~mm}$, there was no incidence of paraplegia. In a series of 7 patients, they had six grafts where the distal landing zone was between T8 and T10. As per EUROSTAR Registry, coverage of T10, or use of greater than two stent-grafts in DTA was significantly associated with spinal cord injury (SCI) (8).

\section{$S C I$}

As per the International E Vita Open Registry database of
274 patients published by the vascular domain of EACTS in 2015, the incidence of SCI after FET was 8\% (9).

On multivariate regression analysis in a report from EUROSTAR Registry, four factors were found to be significant for SCI after FET: (I) greater than two stentgrafts; (II) concomitant open abdominal surgery; (III) renal failure; and (IV) LSA coverage without revascularization (8). They described neurological complications (paraplegia and stroke) in $8.4 \%$ of patients if LSA was covered without revascularization, while zero patient had these complications if LSA was revascularized. For paraplegia alone, LSA coverage without revascularization was a risk factor with an odds ratio of 3.9. In their series of 457 consecutive patients, using Talent stent graft for EVAR, Fattori et al. found LSA occlusion as a risk factor for perioperative stroke, and graft length greater than $20 \mathrm{~cm}$ as a risk factor for paraplegia (10).

Association of renal failure with SCI is probably a surrogate for an underlying metabolic syndrome causing peripheral atherosclerosis, which can compromise the collateral flow to the spinal cord.

Infrarenal aortic replacement as a risk factor for SCI can probably be explained by a study by Khoynezhad et al. (11), in which they found a significant association between occlusion/coverage of hypogastric artery and paraplegia.

Several studies have shown that peri-operative mean arterial blood pressure below $70 \mathrm{mmHg}$ is a risk factor for SCI. CSF drainage with a target CSF pressure less than $10 \mathrm{mmHg}$, especially when a long segment of thoracic aorta is covered with stent-graft, has been found to be protective (12).

\section{Aortic remodeling}

Park et al. (13) in their series of 122 patients undergoing conventional repair for TAAD showed that during the mean follow-up of 33.6 months, aneurysmal degeneration of DTA occurred in $59.7 \%$ of a subset of patients when patent false lumen was wider than the true lumen after repair, in $40 \%$ of patients if false lumen is patent but smaller than the true lumen, in $72 \%$ of patients if false lumen is wider but thrombosed, in $7.7 \%$ of patients if false lumen is smaller and thrombosed in the thoracic segment, and in none if false lumen is thrombosed and smaller in the whole segment (complete aortic remodeling). Patent false lumen is a risk factor for aneurysmal degeneration of distal aorta, late reintervention, and poor survival (14). We know that $50-80 \%$ of patients have patent false lumen after conventional repair of TAAD. This holds even after conventional elephant trunk repair. Residual intimal 
tear in the DTA is the cause of patent false lumen after conventional aortic repair. By using a stent-graft in DTA to exclude this tear from the systemic circulation, false lumen obliteration was achieved in $82-85 \%$ of TAAD patients using this technique $(6,15)$. Chen et al. (16) in their series of 172 Marfan Syndrome patients with TAAD undergoing total arch replacement and FET, showed obliteration of false lumen at the time of hospital discharge in $98.7 \%$ of patients at the proximal end of stent, $86.1 \%$ at the distal end of stent, $39 \%$ in the unstented distal thoracic aorta, and $21 \%$ at the level of renal arteries. They also showed continued expansion of distal true lumen and stability of the false lumen in the unstented portion during follow up CT scans. A report from the vascular domain of EACTS showed that the average rate of early ( $<3$ months) false lumen thrombosis at the level of the stent to be $85 \%$. This number increased to $89 \%$ within 6-12 months of surgery, which shows that aorta continues to remodel over time (9). Vallabhajosyula et al. in a study comparing outcomes after standard hemiarch repair with and without antegrade thoracic endovascular aortic repair (TEVAR), for patients with TAAD presenting with organ malperfusion, reported significantly superior and stable cumulative survival of $68 \%$ at 1 and 5 years after surgery in the subgroup of patients with multi-organ malperfusion and who also underwent antegrade TEVAR in addition to hemiarch replacement. This finding attests to the fact that a stent graft in the DTA deployed in an antegrade manner provides stability and prevents degeneration of the DTA (17). Sultan et al., in their short term follow up study of TAAD patients undergoing conventional open distal hemiarch repair with and without addition of antegrade TEVAR, reported significantly increased true lumen diameter and stable total aortic diameter in the DTA at 12 months with the addition of antegrade TEVAR to conventional repair. Accordingly TEVAR group also showed significantly better false lumen thrombosis of the DTA (18).

\section{Reintervention and survival}

In hospital mortality of $13 \%$ has been reported in patients with TAAD undergoing aggressive hemiarch repair and antegrade stent placement, with a five-year actuarial survival of $73 \pm 8.3$ years (15). Shrestha et al., in their series of 52 patients with TAAD undergoing TEVAR with FET, showed inhospital mortality of $30 \%$, with a stroke rate of $12 \%$ and SCI in $4 \%$ (19). In a follow up of 62 patients using this technique in TAAD patients, the author has previously shown $98 \%$ freedom from open distal aortic intervention at six years (6). In a series of patients with Marfan syndrome undergoing FET for TAAD, the distal reoperation rate was $21 \%$, and the eventfree survival rate was $65 \%$ at ten years (16).

\section{Malperfusion}

As per the German Registry, 33.6\% of TAAD patients present with malperfusion of at least one organ system (5). According to the IRAD database, a subset of TAAD patients with mesenteric malperfusion has an in-hospital mortality of $63.2 \%$. Even after open/hybrid procedure, in-hospital mortality is $41.7 \%$. In a subgroup analysis of patients presenting with multi-organ malperfusion and treated with hemiarch and antegrade TEVAR, in-hospital mortality was down to $28 \%$ (17). In-hospital mortality was $58 \%$ in the conventional hemiarch repair group. They also showed a survival benefit in these patients with multi-organ malperfusion, with a five-year survival of $68 \%$ in the stented group compared to $32 \%$ in patients with conventional repair (17).

\section{Comment}

In a study by Geirsson et al., freedom from reoperation at ten years for TAAD patients undergoing conventional repair was $75.4 \%$ (20). In this study, DeBakey type 1 dissection and Marfan syndrome were the risk factors for distal aortic reintervention. Residual intimal tear in the distal aorta with subsequent patent false lumen is the primary cause of distal aortic degeneration. Distal aortic reoperation carries interval mortality of $32-50 \%$ and in-hospital mortality of $15 \%$.

Conventional elephant trunk can facilitate second distal aortic operation but is unable to prevent or reduce the rate of aortic degeneration. With tears in the distal aortic arch and proximal DTA, elephant trunk has the potential of not expanding completely due to persistent high pressure in the false lumen. In TAAD patients presenting with malperfusion, in-hospital mortality was not found to be significantly affected by the addition of conventional ET, because it fails to expand the true lumen completely unlike FET.

Stent grafting the DTA has the potential to achieve early false lumen thrombosis at the level of graft in $85 \%$ of patients, which increases to $89 \%$ by the end of the first year after surgery. This gives more stability to the aorta and prevents distal aortic degeneration, as evidenced by increased freedom from DTA reintervention. In patients presenting with multiorgan malperfusion, FET by reestablishing flow, the true 
lumen brings down in-hospital mortality significantly.

On the other hand, stent in the DTA provides a stable platform for future endovascular repair if required. If an open distal aortic operation is needed, these stents can be safely clamped. Nitinol stents have an excellent memory and bounce back to their original shape when unclamped.

Fixing the stent graft circumferentially just distal to the LSA prevents any type Ia endoleak. We believe this also prevents flow in the false lumen from entry tear in the brachiocephalic vessels. Gentle oversizing of the stent-graft should decrease the chances of type Ib endoleak. However, our patients are cooled to 15 to $20^{\circ} \mathrm{C}$, which can impact the full expansion of the nitinol frame in stent-grafts and can cause type Ib endoleak. Aggressive oversizing of the stent graft can cause entry tears at the distal landing zone, especially in patients with connective tissue disorders. We do not use antegrade stent graft in DTA if CT images are not available for review before surgery.

Direct repair of intimal tears in brachiocephalic arteries ostium or greater curvature of the arch, with interrupted "inside out" pledgeted suture provides adequate stability to the arch, adequately seal the entry point, and allows doing a hemiarch operation. Total arch replacement for TAAD, although is comparable to hemiarch in the short term, portends an inferior five-year unadjusted survival, and permanent neurological injury rate (21).

We reconstruct proximal and distal aorta with "Teflon felt neo-media," as we believe it stabilizes the aorta and prevents degeneration due to its pro-fibrotic effect. This also causes scarring of aorta around the felt and helps in aortic remodeling. The thrombogenic nature of Teflon also helps in sealing needle hole through the often fragile tissues and can discourage any residual false lumen flow.

SCI and stroke with FET are associated with empiric coverage of LSA without revascularization. This association underscores the importance of often under-appreciated collateral flow to anterior spinal and basilar arteries from the vertebral artery. Collateral supply from the internal mammary artery through anterior intercostal artery might also be necessary. If coverage of LSA is imperative in an acute setting, a careful review of imaging should be done to rule out any acquired or congenital anomaly of LSA, vertebral, and internal mammary arteries. Keeping perioperative arterial blood pressure greater than $70 \mathrm{mmHg}$, CSF drainage, with target CSF pressure $<10 \mathrm{mmHg}$, if a catheter is present, and avoidance of stent-graft coverage of T10 or lower intercostal artery are also essential steps in avoiding SCI.

\section{Conclusions}

Aggressive hemiarch replacement with the direct repair of intimal tear and antegrade TEVAR for TAAD is a safe technique and provides an optimal opportunity for true lumen expansion, mitigates visceral malperfusion, enhances aortic remodeling, and provides a stable platform for any future distal aortic intervention. By ensuring hemodynamic stability of the patient in the perioperative period, avoiding coverage of long length of DTA, and maintaining antegrade flow in LSA, we can keep the incidence of stroke or SCI comparable to that of conventional hemiarch repair, or standard elephant trunk repair.

\section{Acknowledgments}

Funding: None.

\section{Footnote}

Provenance and Peer Review: This article was commissioned by the Guest Editors (Ibrahim Sultan and George Arnaoutakis) for the series "Advancement in the Surgical Treatment of Aortic Dissection" published in Journal of Visualized Surgery. The article has undergone external peer review.

Conflicts of Interest: All authors have completed the ICMJE uniform disclosure form (available at https://jovs. amegroups.com/article/view/10.21037/jovs-20-85/coif). The series "Advancement in the Surgical Treatment of Aortic Dissection" was commissioned by the editorial office without any funding or sponsorship. The authors have no other conflicts of interest to declare.

Ethical Statement: The authors are accountable for all aspects of the work in ensuring that questions related to the accuracy or integrity of any part of the work are appropriately investigated and resolved. All procedures performed in this study were in accordance with the Helsinki Declaration (as revised in 2013). The manuscript is waived from patient informed consent according to the ethics committee or institutional review board.

Open Access Statement: This is an Open Access article distributed in accordance with the Creative Commons Attribution-NonCommercial-NoDerivs 4.0 International License (CC BY-NC-ND 4.0), which permits the noncommercial replication and distribution of the article with 
the strict proviso that no changes or edits are made and the original work is properly cited (including links to both the formal publication through the relevant DOI and the license). See: https://creativecommons.org/licenses/by-nc-nd/4.0/.

\section{References}

1. Hagan PG, Nienaber CA, Isselbacher EM, et al. The International Registry of Acute Aortic Dissection (IRAD): new insights into an old disease. JAMA 2000;283:897-903.

2. Evangelista A, Isselbacher EM, Bossone E, et al. Insights From the International Registry of Acute Aortic Dissection: A 20-Year Experience of Collaborative Clinical Research. Circulation 2018;137:1846-60.

3. Morris GC Jr, Henley WS, Debakey ME. Correction of acute dissecting aneurysm of aorta with valvular insufficiency. JAMA 1963;184:185-6.

4. De Bakey ME, Cooley DA, Creech O Jr. Surgical considerations of dissecting aneurysm of the aorta. Ann Surg 1955;142:586-610; discussion, 611-2.

5. Czerny M, Schoenhoff F, Etz C, et al. The Impact of PreOperative Malperfusion on Outcome in Acute Type A Aortic Dissection: Results From the GERAADA Registry. J Am Coll Cardiol 2015;65:2628-35.

6. Vallabhajosyula P, Szeto WY, Pulsipher A, et al. Antegrade thoracic stent grafting during repair of acute Debakey type I dissection promotes distal aortic remodeling and reduces late open distal reoperation rate. J Thorac Cardiovasc Surg 2014;147:942-8.

7. Faure EM, El Batti S, Abou Rjeili M, et al. Stent-assisted, balloon-induced intimal disruption and relamination of aortic dissection in patients with Marfan syndrome: Midterm outcomes and aortic remodeling. J Thorac Cardiovasc Surg 2018;156:1787-93.

8. Buth J, Harris PL, Hobo R, et al. Neurologic complications associated with endovascular repair of thoracic aortic pathology: Incidence and risk factors. a study from the European Collaborators on Stent/Graft Techniques for Aortic Aneurysm Repair (EUROSTAR) registry. J Vasc Surg 2007;46:1103-10; discussion 1110-1.

9. Shrestha M, Bachet J, Bavaria J, et al. Current status and recommendations for use of the frozen elephant trunk technique: a position paper by the Vascular Domain of EACTS. Eur J Cardiothorac Surg 2015;47:759-69.

10. Fattori R, Nienaber CA, Rousseau H, et al. Results of endovascular repair of the thoracic aorta with the Talent Thoracic stent graft: the Talent Thoracic Retrospective Registry. J Thorac Cardiovasc Surg 2006;132:332-9.
11. Khoynezhad A, Donayre CE, Bui H, et al. Risk factors of neurologic deficit after thoracic aortic endografting. Ann Thorac Surg 2007;83:S882-9; discussion S890-2.

12. Chiesa R, Melissano G, Marrocco-Trischitta MM, et al. Spinal cord ischemia after elective stent-graft repair of the thoracic aorta. J Vasc Surg 2005;42:11-7.

13. Park KH, Lim C, Choi JH, et al. Midterm change of descending aortic false lumen after repair of acute type I dissection. Ann Thorac Surg 2009;87:103-8.

14. Fattouch K, Sampognaro R, Navarra E, et al. Long-term results after repair of type a acute aortic dissection according to false lumen patency. Ann Thorac Surg 2009;88:1244-50.

15. Vallabhajosyula P, Gottret JP, Robb JD, et al. Hemiarch replacement with concomitant antegrade stent grafting of the descending thoracic aorta versus total arch replacement for treatment of acute DeBakey I aortic dissection with arch tear. Eur J Cardiothorac Surg 2016;49:1256-61; discussion 1261.

16. Chen Y, Ma WG, Zhi AH, et al. Fate of distal aorta after frozen elephant trunk and total arch replacement for type A aortic dissection in Marfan syndrome. J Thorac Cardiovasc Surg 2019;157:835-49.

17. Vallabhajosyula P, Gottret JP, Menon R, et al. Central Repair With Antegrade TEVAR for Malperfusion Syndromes in Acute Debakey I Aortic Dissection. Ann Thorac Surg 2017;103:748-55.

18. Sultan I, Wallen TJ, Habertheuer A, et al. Concomitant antegrade stent grafting of the descending thoracic aorta during transverse hemiarch reconstruction for acute DeBakey I aortic dissection repair improves aortic remodeling. J Card Surg 2017;32:581-92.

19. Shrestha M, Fleissner F, Ius F, et al. Total aortic arch replacement with frozen elephant trunk in acute type A aortic dissections: are we pushing the limits too far? †. Eur J Cardiothorac Surg 2015;47:361-6; discussion 366.

20. Geirsson A, Bavaria JE, Swarr D, et al. Fate of the residual distal and proximal aorta after acute type A dissection repair using a contemporary surgical reconstruction algorithm. Ann Thorac Surg 2007;84:1955-64.

21. Kim JB, Chung CH, Moon DH, et al. Total arch repair versus hemiarch repair in the management of acute DeBakey type I aortic dissection. Eur J Cardiothorac Surg 2011;40:881-7.

doi: 10.21037/jovs-20-85

Cite this article as: Singh S, Yousef S, Assi R, Vallabhajosyula P. Frozen elephant trunk and hemiarch for type A aortic dissection. J Vis Surg 2021;7:43. 